\title{
A Note on Nörlund-Type Twisted $q$-Euler Polynomials and Numbers of Higher Order Associated with Fermionic Invariant $q$-Integrals
}

\author{
Leechae Jang \\ Department of Mathematics and Computer Science, KonKuk University, Seol 143-701, Republic of Korea \\ Correspondence should be addressed to Leechae Jang, leechae.jang@kku.ac.kr
}

Received 4 April 2010; Accepted 20 June 2010

Academic Editor: Vijay Gupta

Copyright (c) 2010 Leechae Jang. This is an open access article distributed under the Creative Commons Attribution License, which permits unrestricted use, distribution, and reproduction in any medium, provided the original work is properly cited.

We define multiple Nörlund-type twisted $q$-Euler polynomials and numbers and give interpolation functions of multiple Nörlund-type twisted $q$-Euler polynomials at negative integers. Furthermore, we investigate some identities related to these polynomials and interpolation functions.

\section{Introduction}

Let $p$ be a fixed odd prime number. Throughout this paper, $\mathbb{Z}_{p}, \mathbb{Q}_{p}$, and $\mathbb{C}_{p}$ will be, respectively, the ring of $p$-adic rational integers, the field of $p$-adic rational numbers, and the $p$-adic completion of the algebraic closure of $\mathbb{Q}_{p}$. The $p$-adic absolute value in $\mathbb{C}_{p}$ is normalized so that $|p|_{p}=1 / p$. When one talks of $q$-extension, $q$ is variously considered as an indeterminate, a complex number $q \in \mathbb{C}$, or a $p$-adic number $q \in \mathbb{C}_{p}$. If $q \in \mathbb{C}$, one normally assumes $|q|<1$. If $q \in \mathbb{C}_{p}$, one normally assumes $|1-q|_{p}<p^{1 /(1-p)}$ so that $q^{x}=\exp (x \log q)$ for each $x \in \mathbb{Z}_{p}$. We use the notation

$$
[x]_{q}=\frac{1-q^{x}}{1-q}, \quad[x]_{-q}=\frac{1-(-q)^{x}}{1+q}
$$

compared with [1-22], for all $x \in \mathbb{Z}_{p}$. 
For a fixed odd positive integer $d$ with $(p, d)=1$, set

$$
\begin{gathered}
X=X_{d}=\lim _{\overleftarrow{n}} \mathbb{Z} / d p^{n} \mathbb{Z}, \quad X_{1}=\mathbb{Z}_{p}, \\
X^{*}=\bigcup_{\substack{0<a<d p \\
(a, p)=1}}\left(a+d p \mathbb{Z}_{p}\right), \\
a+d p^{n} \mathbb{Z}_{p}=\left\{x \in X \mid x \equiv a\left(\bmod d p^{n}\right)\right\},
\end{gathered}
$$

where $a \in \mathbb{Z}$ lies in $0 \leq a<d p^{n}$. For any $n \in \mathbb{N}$,

$$
\mu_{q}\left(a+d p^{n} \mathbb{Z}_{p}\right)=\frac{q^{a}}{\left[d p^{n}\right]_{q}}
$$

is known to be a distribution on $\mathbb{Z}_{p}$, compared with [1-22].

The $q$-factorial is defined as $[n]_{q} !=[n]_{q}[n-1]_{q} \cdots[2]_{q}[1]_{q}$, and the Gaussian binomial coefficient is also defined by

$$
\left(\begin{array}{l}
n \\
k
\end{array}\right)_{q}=\frac{[n]_{q} !}{[n-k]_{q} ![k]_{q} !}=\frac{[n]_{q}[n-1]_{q} \cdots[n-k+1]_{q}}{[k]_{q} !}
$$

(see $[7,8])$.

Note that

$$
\lim _{q \rightarrow 1}\left(\begin{array}{l}
n \\
k
\end{array}\right)_{q}=\left(\begin{array}{l}
n \\
k
\end{array}\right)=\frac{n !}{(n-k) ! k !}=\frac{n(n-1) \cdots(n-k+1)}{k !} .
$$

From (1.4), we note that

$$
\left(\begin{array}{c}
n+1 \\
k
\end{array}\right)_{q}=\left(\begin{array}{c}
n \\
k-1
\end{array}\right)_{q}+q^{k}\left(\begin{array}{l}
n \\
k
\end{array}\right)_{q}=q^{n-1}\left(\begin{array}{c}
n \\
k-1
\end{array}\right)_{q}+\left(\begin{array}{l}
n \\
k
\end{array}\right)_{q}
$$

(see $[7,8]$ ).

The $q$-binomial formulae are known as

$$
\begin{gathered}
(b: q)_{n}=(1-b)(1-b q) \cdots\left(1-b q^{n-1}\right)=\sum_{i=0}^{n}\left(\begin{array}{c}
n \\
i
\end{array}\right) q_{q}^{\left(\begin{array}{c}
\left(\begin{array}{l}
2 \\
2
\end{array}\right) \\
q
\end{array}(-1)^{i} b^{i}\right.} \\
\frac{1}{(b: q)_{n}}=\frac{1}{(1-b)(1-b q) \cdots\left(1-b q^{n-1}\right)}=\sum_{i=0}^{\infty}\left(\begin{array}{c}
n+i-1 \\
i
\end{array}\right)_{q} b^{i}
\end{gathered}
$$


The Euler number $E_{n}$ and polynomials $E_{n}(x)$ are defined by the generating function in the complex number field as

$$
\begin{gathered}
\frac{2}{e^{t}+1}=\sum_{n=0}^{\infty} E_{n} \frac{t^{n}}{n !} \quad(|t|<\pi), \\
\frac{2}{e^{t}+1} e^{x t}=\sum_{n=0}^{\infty} E_{n}(x) \frac{t^{n}}{n !} \quad(|t|<\pi) .
\end{gathered}
$$

The $n$th $q$-Euler numbers $E_{n, q}$ and the $n$th $q$-Euler polynomials $E_{n, q}(x)$ attached to $q$ are defined by the exponential generating functions as

$$
\begin{gathered}
F_{q}(t)=2 \sum_{k=0}^{\infty}(-1)^{k} e^{[k]_{q} t}=\sum_{n=0}^{\infty} E_{n, q} \frac{t^{n}}{n !}, \\
F_{q}(t, x)=2 \sum_{k=0}^{\infty}(-1)^{k} e^{[k+x]_{q} t}=\sum_{n=0}^{\infty} E_{n, q}(x) \frac{t^{n}}{n !} .
\end{gathered}
$$

The $n$th Euler numbers $E_{n}^{(r)}$ of higher order and the $n$th Euler polynomials $E_{n}^{(r)}(x)$ of higher order attached to $q$ are defined by the exponential generating functions as

$$
\begin{gathered}
F^{(r)}(t)=\frac{2^{r}}{\left(1+e^{t}\right)^{r}}=\sum_{k=0}^{\infty} E_{n}^{(r)} \frac{t^{n}}{n !}, \\
F^{(r)}(t, x)=\frac{2^{r}}{\left(1+e^{t}\right)^{r}} e^{x t}=\sum_{k=0}^{\infty} E_{n}^{(r)}(x) \frac{t^{n}}{n !}, \\
F^{(-r)}(t, x)=\frac{\left(1+e^{t}\right)^{r}}{2^{r}} e^{x t}=\sum_{k=0}^{\infty} E_{n}^{(-r)}(x) \frac{t^{n}}{n !} .
\end{gathered}
$$

Kim [7] defined the $n$th $q$-Euler numbers $E_{n, q}^{(r)}$ of higher order, the Euler polynomials $E_{n, q}^{(r)}(x)$ of higher order, and the $n$th Nörlund-type $q$-Euler polynomials of higher order which are defined by the exponential generating functions as

$$
\begin{gathered}
F_{q}^{(r)}(t)=2^{r} \sum_{m=0}^{\infty}(-1)^{m}\left(\begin{array}{c}
m+r-1 \\
m
\end{array}\right) e_{q} e^{[m]_{q} t}=\sum_{n=0}^{\infty} E_{n, q}^{(r)} \frac{t^{n}}{n !}, \\
F_{q}^{(r)}(t, x)=2^{r} \sum_{m=0}^{\infty}(-1)^{m}\left(\begin{array}{c}
m+r-1 \\
m
\end{array}\right)_{q} e^{[m+x]_{q} t}=\sum_{n=0}^{\infty} E_{n, q}^{(r)}(x) \frac{t^{n}}{n !} \\
F_{q}^{(-r)}(t, x)=\sum_{n=0}^{\infty} E_{n, q}^{(-r)}(x) \frac{t^{n}}{n !},
\end{gathered}
$$

compared with [6-16, 18-21]. 
We say that $f$ is uniformly differentiable function at a point $a \in \mathbb{Z}_{p}$ and denote this property by $f \in \mathrm{UD}\left(\mathbb{Z}_{p}\right)$ if the difference quotients

$$
F_{f}(x, y)=\frac{f(x)-f(y)}{x-y}
$$

have a limit $l=f^{\prime}(a)$ as $(x, y) \rightarrow(a, a)$, compared with [1-22] (23-24). Note that the bosonic $p$-adic $q$-integral of a function $f \in \operatorname{UD}\left(\mathbb{Z}_{p}\right)$ was defined by

$$
I_{q}(f)=\int_{\mathbb{Z}_{p}} f(x) d \mu_{q}(x)=\lim _{n \rightarrow \infty} \frac{1}{\left[p^{n}\right]_{q}} \sum_{x=0}^{p^{n}-1} f(x) q^{x}
$$

and that the fermionic $p$-adic $q$-integral was defined by

$$
I_{-q}(f)=\int_{\mathbb{Z}_{p}} f(x) d \mu_{-q}(x)=\lim _{n \rightarrow \infty} \frac{1}{\left[p^{n}\right]_{-q}} \sum_{x=0}^{p^{n}-1} f(x)(-q)^{x}
$$

(see [1-22] (23-24) ). In (1.15), when $q \rightarrow 1$, we can obtain

$$
L_{-1}\left(f_{1}\right)+L_{-1}(f)=2 f(0)
$$

where $f_{1}(x)=f(x+1)$. If we take $f(x)=e^{t x}$, then we obtain

$$
I_{-1}\left(e^{t x}\right)=\int_{\mathbb{Z}_{p}} e^{t x} d \mu_{-1}(x)=\frac{2}{e^{t}+1}
$$

In this paper, we define multiple Nörlund-type twisted $q$-Euler polynomials and give interpolation functions of multiple Nörlund-type twisted $q$-Euler polynomials at negative integers. Furthermore, we investigate some identities related to these polynomials and interpolation functions. 


\section{Nörlund-Type Twisted $q$-Euler Numbers and Polynomials of Higher Order}

In this section, we assume that $q \in \mathbb{C}_{p}$ with $|1-q|_{p}<1$. Let $C_{p^{\infty}}=\bigcup_{n \geq 1} C_{p^{n}}=\lim _{n \rightarrow \infty} C_{p^{n}}$ be the locally constant space, when $C_{p^{n}}=\left\{\xi \in X \mid \xi^{p^{n}}=1\right\}$ is the cyclic group of order $p^{n}$. Let $\xi \in C_{p^{\infty}}$. We define the twisted $q$-Euler polynomials (see $[1-5,18-21]$ ) as follows:

$$
\begin{aligned}
E_{m, q, \xi}^{(r)}(x) & =\int_{\mathbb{Z}_{p}} \cdots \int_{\mathbb{Z}_{p}}\left[x+x_{1}+\cdots+x_{r}\right]_{q}^{n} \xi^{x_{1}+\cdots+x_{r}} d \mu_{-1}\left(x_{1}\right) \cdots d \mu_{-1}\left(x_{r}\right) \\
& =\frac{2^{r}}{(1-q)^{n}} \sum_{l=0}^{n}\left(\begin{array}{c}
n \\
l
\end{array}\right)(-1)^{l} q^{l x}\left(\frac{1}{1+q^{l} \xi^{l}}\right)^{r} \\
& =2^{r} \sum_{m=0}^{\infty}\left(\begin{array}{c}
m+r-1 \\
m
\end{array}\right)(-1)^{m}[m+x]_{q, \xi^{m}}^{n},
\end{aligned}
$$

where $[a]_{q, \xi}=\left(1-q^{a} \xi\right) /(1-q)$. Let $F_{q, \xi}^{(r)}(t, x)=\sum_{n=0}^{\infty} E_{n, q, \xi}^{(r)}(x)\left(t^{n} / n !\right)$. Then we have

$$
F_{q, \xi}^{(r)}(t, x)=2^{r} \sum_{m=0}^{\infty}\left(\begin{array}{c}
m+r-1 \\
m
\end{array}\right)(-1)^{m} e^{[m+x]_{q, \xi} t}
$$

In this special case $x=0, E_{n, q, \xi}^{(r)}(0)=E_{n, q, \xi}^{(r)}$ are called the twisted $q$-Euler numbers of order $r$. In the sense of the twisted in $(1.11-1)$, we consider the Nörlund-type twisted $q$-Euler polynomials as follows:

$$
G_{q, \xi}^{(r)}(t, x)=F_{q, \xi}^{(-r)}(t, x)=\frac{1}{2^{r}} \sum_{m=0}^{r}\left(\begin{array}{c}
r \\
m
\end{array}\right) e^{[m+x]_{q, \xi^{m}} t}=\sum_{n=0}^{\infty} E_{n, q, \xi}^{(-r)}(x) \frac{t^{n}}{n !}
$$

By (2.3), we have

$$
E_{n, q, \xi}^{(-r)}(x)=\frac{1}{2^{r}} \sum_{m=0}^{r}\left(\begin{array}{c}
r \\
m
\end{array}\right)[m+x]_{q, \xi^{m}}^{n}
$$

From (2.1) and (2.4), we can obtain the following theorem.

Theorem 2.1. For $r \in \mathbb{N}, n \geq 0$, and $\varepsilon \in T_{p}$, let

$$
2^{r} \sum_{m=0}^{\infty}\left(\begin{array}{c}
m+r-1 \\
m
\end{array}\right)(-\xi)^{m} e^{[m+x]_{q, \xi^{m}} t}=\sum_{n=0}^{\infty} E_{n, q, \xi}^{(r)}(x) \frac{t^{n}}{n !} .
$$


Then

$$
\begin{aligned}
E_{n, q, \xi}^{(r)}(x) & =\frac{2^{r}}{(1-q)^{n}} \sum_{l=0}^{n}\left(\begin{array}{c}
n \\
l
\end{array}\right)(-1)^{l} q^{l x}\left(\frac{1}{1+q^{l} \xi^{l}}\right)^{r} \\
& =2^{r} \sum_{m=0}^{\infty}\left(\begin{array}{c}
m+r-1 \\
m
\end{array}\right)(-1)^{m}[m+x]_{q, \xi^{m}}^{n}, \\
E_{n, q, \xi}^{(-r)}(x) & =\frac{1}{2^{r}(1-q)^{n}} \sum_{l=0}^{n}\left(\begin{array}{c}
n \\
l
\end{array}\right) q^{l x}\left(1+q^{l} \xi^{l}\right)^{r} \\
& =\frac{1}{2^{r}} \sum_{m=0}^{r}\left(\begin{array}{c}
r \\
m
\end{array}\right)[m+x]_{q, \xi^{m}}^{n} .
\end{aligned}
$$

$E_{n, q, \xi}^{(-r)}(0)=E_{n, q, \xi}^{(-r)}$ are called the twisted $q$-Euler numbers of higher order. For $h \in \mathbb{Z}$, $r \in \mathbb{N}$, let us define the twisted $q$-Euler polynomials of higher order as follows:

$$
E_{n, q, \xi}^{(h, r)}(x)=\int_{\mathbb{Z}_{p}} \cdots \int_{\mathbb{Z}_{p}} q^{\sum_{j=1}^{r}(h-j) x_{j}} \xi^{\sum_{j=1}^{r} x_{j}}\left[x+x_{1}+\cdots+x_{r}\right]_{q}^{n} d \mu_{-1}\left(x_{1}\right) \cdots d \mu_{-1}\left(x_{r}\right) .
$$

Then

$$
\begin{aligned}
E_{n, q, \xi}^{(h, r)}(x) & =\frac{2^{r}}{(1-q)^{n}} \sum_{l=0}^{n} \frac{\left(\begin{array}{c}
n \\
l
\end{array}\right)(-1)^{l} q^{l x}}{\left(1 /\left(1+q^{l} \xi^{l}\right)\right)_{r}} \\
& =\frac{2^{r}}{(1-q)^{n}} \sum_{l=0}^{n} \frac{\left(\begin{array}{c}
n \\
l
\end{array}\right)(-1)^{l} q^{l x}}{\left(-q^{h-1+l} \xi^{l} ; q^{-1}\right)_{r}} \\
& =2^{r} \sum_{m=0}^{\infty}\left(\begin{array}{c}
m+r-1 \\
m
\end{array}\right) q_{q}^{(h-r) m}(-1)^{m}[x+m]_{q, \xi^{m}}^{n} .
\end{aligned}
$$

Let $F_{q, \xi}^{(h, r)}(t, x)=\sum_{m=0}^{\infty} E_{n, q, \xi}^{(h, r)}\left(x_{1}\right)\left(t^{n} / n !\right)$. By (2.7), we easily see that

$$
E_{n, q, \xi}^{(h, r)}(x)=\int_{\mathbb{Z}_{p}} \cdots \int_{\mathbb{Z}_{p}} q^{\sum_{j=1}^{r}(h-j) x_{j}} \xi^{\sum_{j=1}^{r} x_{j}}\left[x+x_{1}+\cdots+x_{r}\right]_{q}^{n} d \mu_{-1}\left(x_{1}\right) \cdots d \mu_{-1}\left(x_{r}\right) .
$$

Thus, we obtain the following theorem.

Theorem 2.2. For $h \in \mathbb{Z}, r \in \mathbb{N}, n \geq 0$, and $\varepsilon \in T_{p}$, let

$$
2^{r} \sum_{m=0}^{\infty}\left(\begin{array}{c}
m+r-1 \\
m
\end{array}\right) q_{q}^{(h-r) m}(-1)^{m} e^{[m+x]_{q, \xi^{m}} t}=\sum_{n=0}^{\infty} E_{n, q, \xi}^{(h, r)}(x) \frac{t^{n}}{n !} .
$$


Then

$$
\begin{aligned}
E_{n, q, \xi}^{(h, r)}(x) & =\frac{2^{r}}{(1-q)^{n}} \sum_{l=0}^{n} \frac{\left(\begin{array}{c}
n \\
l
\end{array}\right)(-1)^{l} q^{l x}}{\left(-q h^{h-r+l} ; q\right)_{r}} \\
& =2^{r} \sum_{m=0}^{\infty} q^{(h-r) m}(-1)^{m}\left(\begin{array}{c}
m+r-1 \\
m
\end{array}\right)_{q}(-1)^{m}[m+x]_{q, \xi^{m}}^{n}
\end{aligned}
$$
follows:

Now, we define the Nörlund-type twisted $q$-Euler polynomials of higher order as

$$
E_{n, q, \xi}^{(h,-r)}(x)=\frac{1}{(1-q)^{n}} \sum_{l=0}^{n} \frac{\left(\begin{array}{c}
n \\
l
\end{array}\right)(-1)^{l} q^{l x}}{\int_{\mathbb{Z}_{p}} \cdots \int_{\mathbb{Z}_{p}} q^{l\left(x_{1}+\cdots+x_{r}\right)} q^{\sum_{j=1}^{r}(h-j) x_{j}} \xi^{l\left(x_{1}+\cdots+x_{r}\right)} d \mu_{-1}\left(x_{1}\right) \cdots d \mu_{-1}\left(x_{r}\right)} .
$$

Let $F_{q, \xi}^{(h,-r)}(t, x)=\sum_{m=0}^{\infty} E_{n, q, \xi}^{(h,-r)}\left(x_{1}\right)\left(t^{n} / n !\right)$. By (2.12), we have

$$
F_{q, \xi}^{(h,-r)}(t, x)=\frac{1}{2^{r}} \sum_{m=0}^{r} q^{\left(\begin{array}{c}
m \\
2
\end{array}\right)} q^{(h-r) m}\left(\begin{array}{c}
r \\
m
\end{array}\right)_{q} e^{[m+x]_{q, \xi^{m}} t}
$$

Thus, we obtain the following theorem.

Theorem 2.3. For $h \in \mathbb{Z}, r \in \mathbb{N}, n \geq 0$, and $\varepsilon \in T_{p}$, let

$$
\frac{1}{2^{r}} \sum_{m=0}^{r} q^{\left(\begin{array}{c}
m \\
2
\end{array}\right)} q^{(h-r) m}\left(\begin{array}{c}
r \\
m
\end{array}\right){ }_{q} e^{[m+x]_{q, \xi^{m}} t}=\sum_{n=0}^{\infty} E_{n, q, \xi}^{(h,-r)}(x) \frac{t^{n}}{n !} .
$$

Then

$$
\begin{aligned}
E_{n, q, \xi}^{(h,-r)}(x) & =\frac{1}{2^{r}(1-q)^{n}} \sum_{l=0}^{n}\left(\begin{array}{c}
n \\
l
\end{array}\right)(-1)^{l} q^{l x}\left(-q^{h-r+l} \xi ; q\right)_{r} \\
& =\frac{1}{2^{r}} \sum_{m=0}^{r} q^{\left(\frac{m}{2}\right)} q^{(h-r) m}\left(\begin{array}{c}
r \\
m
\end{array}\right)_{q}[m+x]_{q, \xi^{m}}^{n} .
\end{aligned}
$$


For $h=r$, we have

$$
\begin{aligned}
E_{n, q, \xi}^{(r, r)}(x) & =\frac{2^{r}}{(1-q)^{n}} \sum_{l=0}^{n} \frac{\left(\begin{array}{c}
n \\
l
\end{array}\right)(-1)^{l} q^{l x}}{\left(-q h^{l} ; q\right)_{r}} \\
& =2^{r} \sum_{m=0}^{\infty}(-1)^{m}\left(\begin{array}{c}
m+r-1 \\
m
\end{array}\right)_{q}(-1)^{m}[m+x]_{q, \xi^{m}}^{n}, \\
E_{n, q, \xi}^{(r,-r)}(x) & =\frac{1}{2^{r}(1-q)^{n}} \sum_{l=0}^{n}\left(\begin{array}{c}
n \\
l
\end{array}\right)(-1)^{l} q^{l x}\left(-q^{l} \xi^{l} ; q\right)_{r} \\
& =\frac{1}{2^{r}} \sum_{m=0}^{r} q^{\left(\begin{array}{c}
m \\
2
\end{array}\right)} q^{(h-r) m}\left(\begin{array}{c}
r \\
m
\end{array}\right)_{q}[m+x]_{q, \xi^{m}}^{n} .
\end{aligned}
$$

Thus, it is easy to see that

$$
\begin{aligned}
\frac{q^{m x} 2^{r}}{\left(-q^{m-r l} ; q\right)_{r}} & =\int_{\mathbb{Z}_{p}} \cdots \int_{\mathbb{Z}_{p}} q^{\sum_{j=1}^{r}(m-j) x_{j}+m x} \xi^{\sum_{j=1}^{r} x_{j}} d \mu_{-1}\left(x_{1}\right) \cdots d \mu_{-1}\left(x_{r}\right) \\
& =\int_{\mathbb{Z}_{p}} \cdots \int_{\mathbb{Z}_{p}}\left(\left[x+x_{1}+\cdots+x_{r}\right]_{q}(q-1)+1\right)^{m} q^{\sum_{j=1}^{r} x_{j}} \xi^{\sum_{j=1}^{r} x_{j}} d \mu_{-1}\left(x_{1}\right) \cdots d \mu_{-1}\left(x_{r}\right) \\
& =\sum_{l=0}^{m}\left(\begin{array}{c}
m \\
l
\end{array}\right)(q-1)^{l} \int_{\mathbb{Z}_{p}} \cdots \int_{\mathbb{Z}_{p}}\left[x+x_{1}+\cdots+x_{r}\right]_{q}^{l} q^{\sum_{j=1}^{r} x_{j}} \xi^{\sum_{j=1}^{r} x_{j}} d \mu_{-1}\left(x_{1}\right) \cdots d \mu_{-1}\left(x_{r}\right) \\
& =\sum_{l=0}^{m}\left(\begin{array}{c}
m \\
l
\end{array}\right)(q-1)^{l} E_{l, q, \xi}^{(0, r)}(x) .
\end{aligned}
$$

Equation (2.18) implies that

$$
\frac{q^{m x} 2^{r}}{\left(-q^{m-r} \xi ; q\right)_{r}}=\sum_{l=0}^{m}\left(\begin{array}{c}
m \\
l
\end{array}\right)(q-1)^{l} E_{l, q, \xi}^{(0, r)}(x) .
$$

From (1.16), we derive

$$
\begin{aligned}
q^{h-1} \int_{\mathbb{Z}_{p}} \cdots \int_{\mathbb{Z}_{p}}\left[x+1+x_{1}+\cdots+x_{r}\right]_{q}^{m} q^{\sum_{j=1}^{r}(h-j) x_{j}} \xi^{\sum_{j=1}^{r} x_{j}} d \mu_{-1}\left(x_{1}\right) \cdots d \mu_{-1}\left(x_{r}\right) \\
=-\int_{\mathbb{Z}_{p}} \cdots \int_{\mathbb{Z}_{p}}\left[x+1+x_{1}+\cdots+x_{r}\right]_{q}^{m} q^{\sum_{j=1}^{r}(h-j) x_{j}} \xi^{\sum_{j=1}^{r} x_{j}} d \mu_{-1}\left(x_{1}\right) \cdots d \mu_{-1}\left(x_{r}\right) \\
\quad+2 \int_{\mathbb{Z}_{p}} \cdots \int_{\mathbb{Z}_{p}}\left[x+1+x_{1}+\cdots+x_{r}\right]_{q}^{m} q^{\sum_{j=1}^{r-1}(h-1-j) x_{j+1}} \xi^{\sum_{j=1}^{r-1} x_{j+1}} d \mu_{-1}\left(x_{1}\right) \cdots d \mu_{-1}\left(x_{r}\right) .
\end{aligned}
$$


By (2.20), we have

$$
q^{h-1} E_{n, q, \xi}^{(h, r)}(x+1)+E_{n, q, \xi}^{(h, r)}(x)=2 E_{n, q, \xi}^{(h-1, r-1)}(x) .
$$

By simple calculation, we see that

$$
\begin{aligned}
q^{x} \int_{\mathbb{Z}_{p}} & \cdots \int_{\mathbb{Z}_{p}}\left[x_{1}+\cdots+x_{r}+x\right]_{q}^{n} q^{\sum_{j=1}^{r}(h-j+1) x_{j}} \xi^{\sum_{j=1}^{r} x_{j}} d \mu_{-1}\left(x_{1}\right) \cdots d \mu_{-1}\left(x_{r}\right) \\
= & (q-1) \int_{\mathbb{Z}_{p}} \cdots \int_{\mathbb{Z}_{p}}\left[x_{1}+\cdots+x_{r}+x\right]_{q}^{n+1} q^{\sum_{j=1}^{r}(h-j) x_{j}} \xi^{\sum_{j=1}^{r} x_{j}} d \mu_{-1}\left(x_{1}\right) \cdots d \mu_{-1}\left(x_{r}\right) \\
& +\int_{\mathbb{Z}_{p}} \cdots \int_{\mathbb{Z}_{p}}\left[x_{1}+\cdots+x_{r}+x\right]_{q}^{m} q^{\sum_{j=1}^{r}(h-j) x_{j}} \xi^{\sum_{j=1}^{r} x_{j}} d \mu_{-1}\left(x_{1}\right) \cdots d \mu_{-1}\left(x_{r}\right) .
\end{aligned}
$$

By (2.22), we see that

$$
q^{x} E_{n, q, \xi}^{(h+1, r)}(x)=(q-1) E_{n+1, q, \xi}^{(h, r)}(x)+E_{n, q, \xi}^{(h, r)}(x) .
$$

Therefore, we obtain the following theorem.

Theorem 2.4. For $h \in \mathbb{Z}, r \in \mathbb{N}, n \geq 0$, and $\varepsilon \in T_{p}$,

$$
\begin{aligned}
& q^{h-1} E_{n, q, \xi}^{(h, r)}(x+1)+E_{n, q, \xi}^{(h, r)}(x)=2 E_{n, q, \xi}^{(h-1, r-1)}(x), \\
& q^{x} E_{n, q, \xi}^{(h+1, r)}(x)=(q-1) E_{n+1, q, \xi}^{(h, r)}(x)+E_{n, q, \xi}^{(h, r)}(x) .
\end{aligned}
$$

Moreover,

$$
\frac{q^{m x} 2^{r}}{\left(-q^{m-r} \xi ; q\right)_{r}}=\sum_{l=0}^{m}\left(\begin{array}{c}
m \\
l
\end{array}\right)(q-1)^{l} E_{l, q, \xi}^{(0, r)}(x)
$$

From (2.16), we note that

$$
\begin{aligned}
E_{n, q^{-1}, \xi}^{(r, r)}(r-x) & =\frac{2^{r}}{\left(1-q^{-1}\right)^{n}} \sum_{l=0}^{n} \frac{\left(\begin{array}{c}
n \\
l
\end{array}\right)(-1)^{l} q^{l(r-x)}}{\left(-q^{-l} \xi ; q^{-1}\right)_{r}} \\
& =(-1)^{n} q^{n+\left(\begin{array}{c}
r \\
2
\end{array}\right)} \frac{2^{r}}{(1-q)^{n}} \sum_{l=0}^{n} \frac{\left(\begin{array}{c}
n \\
l
\end{array}\right)(-1)^{l} q^{l x}}{\left(-q^{l} \xi ; q\right)_{r}} \\
& =(-1)^{n} q^{n+\left(\begin{array}{c}
r \\
2
\end{array}\right)} E_{n, q, \xi}^{(r, r)}(x) .
\end{aligned}
$$

In the case $x=r$, we obtain

$$
E_{n, q^{-1, \xi}}^{(r, r)}(0)=(-1)^{n} q^{n+\left(\begin{array}{c}
r \\
2
\end{array}\right)} E_{n, q, \xi}^{(r, r)}(r)
$$


From (2.21) with $h=r$, we derive

$$
q^{r-1} E_{n, q, \xi}^{(r, r)}(x+1)+E_{n, q, \xi}^{(r, r)}(x)=2 E_{n, q, \xi}^{(r-1, r-1)}(x) .
$$

\section{Further Remarks on Nörlund-Type Twisted $q$-Euler Polynomials}

In the case $h=0$, let us consider the polynomials $E_{n, q, \xi}^{(0, r)}(x)$ and $E_{n, q, \xi}^{(0,-r)}(x)$ as follows:

$$
\begin{gathered}
E_{n, q, \xi}^{(0, r)}(x)=\int_{\mathbb{Z}_{p}} \cdots \int_{\mathbb{Z}_{p}} q^{-\sum_{j=1}^{r} j x_{j}} \xi^{\sum_{j=1}^{r} x_{j}}\left[x+x_{1}+\cdots+x_{r}\right]_{q}^{n} d \mu_{-1}\left(x_{1}\right) \cdots d \mu_{-1}\left(x_{r}\right), \\
E_{n, q, \xi}^{(0,-r)}(x)=\sum_{l=0}^{n} \frac{\left(\begin{array}{c}
n \\
l
\end{array}\right)(-1)^{l} q^{l x}}{\int_{\mathbb{Z}_{p}} \cdots \int_{\mathbb{Z}_{p}} q^{l\left(x_{1}+\cdots+x_{r}\right)} q^{-\sum_{j=1}^{r} j x_{j}} \xi^{l\left(x_{1}+\cdots+x_{r}\right)} d \mu_{-1}\left(x_{1}\right) \cdots d \mu_{-1}\left(x_{r}\right)} .
\end{gathered}
$$

Then we have

$$
\begin{aligned}
& E_{n, q, \xi}^{(0, r)}(x)=\frac{2^{r}}{(1-q)^{n}} \sum_{l=0}^{n} \frac{\left(\begin{array}{c}
n \\
l
\end{array}\right)(-1)^{l} q^{l x}}{\left(-q^{l-r} \xi ; q\right)_{r}} \\
& =2^{r} \sum_{m=0}^{\infty}\left(\begin{array}{c}
m+r-1 \\
m
\end{array}\right)_{q} q^{-r m}(-1)^{m}[x+m]_{q, \xi^{m}}^{n}, \\
& E_{n, q, \xi}^{(0,-r)}(x)=\frac{1}{2^{r}(1-q)^{n}} \sum_{l=0}^{n}\left(\begin{array}{l}
n \\
l
\end{array}\right)(-1)^{l} q^{l x}\left(-q^{l-r} \xi ; q\right)_{r} \\
& =\frac{1}{2^{r}} \sum_{m=0}^{r} q^{\left(\begin{array}{c}
m \\
2
\end{array}\right)} q^{(h-r) m}\left(\begin{array}{c}
r \\
m
\end{array}\right)_{q}[m+x]_{q, \xi}^{n} .
\end{aligned}
$$

Let us consider the following polynomials:

$$
E_{n, q, \xi}^{(h, 1)}(x)=\int_{\mathbb{Z}_{p}} q^{x_{1}(h-1)} \xi^{x_{1}}\left[x+x_{1}\right]_{q}^{n} d \mu_{-1}\left(x_{1}\right)=\frac{2}{(1-q)^{n}} \sum_{l=0}^{n} \frac{\left(\begin{array}{c}
n \\
l
\end{array}\right)(-1)^{l} q^{l x}}{1+q^{l+h-1} \xi^{l}} .
$$

By the simple calculation of fermionic $p$-adic invariant integral, we see that

$$
\begin{aligned}
q^{x} \int_{\mathbb{Z}_{p}} q^{x_{1}(h-1)} \xi^{x_{1}}\left[x+x_{1}\right]_{q}^{n} d \mu_{-1}\left(x_{1}\right)= & (q-1) \int_{\mathbb{Z}_{p}} q^{x_{1}(h-2)} \xi^{x_{1}}\left[x+x_{1}\right]_{q}^{n+1} d \mu_{-1}\left(x_{1}\right) \\
& +\int_{\mathbb{Z}_{p}} q^{x_{1}(h-2)} \xi^{x_{1}}\left[x+x_{1}\right]_{q}^{n} d \mu_{-1}\left(x_{1}\right) .
\end{aligned}
$$

Thus

$$
q^{x} E_{n, q, \xi}^{(h, 1)}(x)=(q-1) E_{n+1, q, \xi}^{(h-1,1)}(x)+E_{n, q, \xi}^{(h-1,1)}(x) .
$$


It is easy to see that

$$
\int_{\mathbb{Z}_{p}} q^{(h-1) x_{1}} \xi^{x_{1}}\left[x+x_{1}\right]_{q}^{n} d \mu_{-1}\left(x_{1}\right)=\sum_{j=1}^{n}\left(\begin{array}{l}
n \\
j
\end{array}\right)[x]_{q}^{n-j} q^{j x} \int_{\mathbb{Z}_{p}}\left[x_{1}\right]_{q}^{j} q^{(h-1) x_{1}} \xi^{x_{1}} d \mu_{-1}\left(x_{1}\right) .
$$

By (2.20), we can obtain that

$$
E_{n, q, \xi}^{(h, 1)}(x)=\sum_{j=0}^{n}\left(\begin{array}{c}
n \\
j
\end{array}\right)[x]_{q}^{n-j} q^{j x} E_{j, q, \xi}^{(h, 1)}=\left(q^{x} E_{q, \xi}^{(h, 1)}+[x]_{q}\right)^{n}, \quad n \geq 0,
$$

where we use the technique method notation by replacing $\left(E_{q, \xi}^{(h, 1)}\right)^{n}$ by $E_{n, q, \xi}^{(h, 1)}$, symbolically. From (1.14), we can also derive

$$
\int_{\mathbb{Z}_{p}} q^{(h-1)\left(x_{1}+1\right)} \xi^{x_{1}+1}\left[x+x_{1}+1\right]_{q}^{n} d \mu_{-1}\left(x_{1}\right)+\int_{\mathbb{Z}_{p}}\left[x+x_{1}\right]_{q}^{n} q^{(h-1) x_{1}} \xi^{x_{1}} d \mu_{-1}\left(x_{1}\right)=2[x]_{q}^{n}
$$

Thus, we obtain that

$$
q^{h-1} E_{n, q, \xi}^{(h, 1)}(x+1)+E_{n, q, \xi}^{(h, 1)}(x)=2[x]_{q}^{n} .
$$

For $x=0$, we have

$$
q^{h-1}\left(E_{n, q, \xi}^{(h, 1)}+1\right)^{n}+E_{n, q, \xi}^{(h, 1)}=2 \delta_{n, 0}
$$

where $\delta_{n, 0}$ is the Kronecker symbol. It is easy to see that

$$
E_{0, q, \xi}^{(h, 1)}=\int_{\mathbb{Z}_{p}} q^{x_{1}(h-1)} \xi^{x_{1}} d \mu_{-1}\left(x_{1}\right)=\frac{2}{1+q^{h-1} \xi}=\frac{2}{[2]_{q^{h-1, \xi}}} .
$$

By (3.3), we see that

$$
\begin{aligned}
E_{n, q, \xi}^{(h, 1)}(1-x) & =\int_{\mathbb{Z}_{p}}\left[1-x+x_{1}\right]_{q-1}^{n} q^{-x_{1}(h-1)} \xi^{x_{1}} d \mu_{-1}\left(x_{1}\right) \\
& =(-1)^{n} q^{n+h-1} \frac{2}{(1-q)^{n}} \sum_{l=0}^{n} \frac{\left(\begin{array}{c}
n \\
l
\end{array}\right)(-1)^{l} q^{l x}}{1+q^{l+h-1} \xi^{l}} \\
& =(-1)^{n} q^{n+h-1} E_{n, q, \xi}^{(h, 1)}(x) .
\end{aligned}
$$

In particular, for $x=1$, we obtain that

$$
E_{n, q, \xi}^{(h, 1)}(0)=(-1)^{n} q^{n+h-1} E_{n, q, \xi}^{(h, 1)}(1)=(-1)^{n-1} q^{n} E_{n, q, \xi}^{(h, 1)} .
$$




\section{Acknowledgment}

This paper was supported by Konkuk University in 2010.

\section{References}

[1] M. Can, M. Cenkci, V. Kurt, and Y. Simsek, "Twisted Dedekind type sums associated with Barnes' type multiple Frobenius-Euler l-functions," Advanced Studies in Contemporary Mathematics, vol. 18, no. 2, pp. 135-160, 2009.

[2] M. Cenkci, "The $p$-adic generalized twisted $(h, q)$-Euler-l-function and its applications," Advanced Studies in Contemporary Mathematics, vol. 15, no. 1, pp. 37-47, 2007.

[3] L.-C. Jang and C.-S. Ryoo, "A note on the multiple twisted Carlitz's type $q$-Bernoulli polynomials," Abstract and Applied Analysis, vol. 2008, Article ID 498173, 7 pages, 2008.

[4] L.-C. Jang, "Multiple twisted $q$-Euler numbers and polynomials associated with $p$-adic $q$-integrals," Advances in Difference Equations, vol. 2008, Article ID 738603, 11 pages, 2008.

[5] L.-C. Jang, "A study on the distribution of twisted $q$-Genocchi polynomials," Advanced Studies in Contemporary Mathematics, vol. 18, no. 2, pp. 181-189, 2009.

[6] T. Kim, " $q$-Volkenborn integration," Russian Journal of Mathematical Physics, vol. 9, no. 3, pp. 288-299, 2002.

[7] T. Kim, "Some identities on the $q$-Euler polynomials of higher order and $q$-Stirling numbers by the fermionic $p$-adic integral on $\mathbb{Z}_{p}, "$ Russian Journal of Mathematical Physics, vol. 16, no. 4, pp. 484-491, 2009.

[8] T. Kim, "Note on the Euler q-zeta functions," Journal of Number Theory, vol. 129, no. 7, pp. 1798-1804, 2009.

[9] T. Kim, "The modified $q$-Euler numbers and polynomials," Advanced Studies in Contemporary Mathematics, vol. 16, no. 2, pp. 161-170, 2008.

[10] T. Kim, "Note on Dedekind type DC sums," Advanced Studies in Contemporary Mathematics, vol. 18, no. 2, pp. 249-260, 2009.

[11] T. Kim, "On Euler-Barnes multiple zeta functions," Russian Journal of Mathematical Physics, vol. 10, no. 3, pp. 261-267, 2003.

[12] T. Kim, "On a $q$-analogue of the $p$-adic log gamma functions and related integrals," Journal of Number Theory, vol. 76, no. 2, pp. 320-329, 1999.

[13] T. Kim, "On the analogs of Euler numbers and polynomials associated with $p$-adic $q$-integral on $\mathbb{Z}_{p}$ at $q=-1, "$ Journal of Mathematical Analysis and Applications, vol. 331, no. 2, pp. 779-792, 2007.

[14] T. Kim, "On $p$-adic interpolating function for $q$-Euler numbers and its derivatives," Journal of Mathematical Analysis and Applications, vol. 339, no. 1, pp. 598-608, 2008.

[15] T. Kim, "On the multiple $q$-Genocchi and Euler numbers," Russian Journal of Mathematical Physics, vol. 15, no. 4, pp. 481-486, 2008.

[16] T. Kim, "Symmetry of power sum polynomials and multivariate fermionic $p$-adic invariant integral on $\mathbb{Z}_{p}$," Russian Journal of Mathematical Physics, vol. 16, no. 1, pp. 93-96, 2009.

[17] Y.-H. Kim, W. Kim, and C. S. Ryoo, "On the twisted $q$-Euler zeta function associated with twisted q-Euler numbers," Proceedings of the Jangjeon Mathematical Society, vol. 12, no. 1, pp. 93-100, 2009.

[18] S.-H. Rim, S. J. Lee, E. J. Moon, and J. H. Jin, "On the $q$-Genocchi numbers and polynomials associated with $q$-zeta function," Proceedings of the Jangjeon Mathematical Society, vol. 12, no. 3, pp. 261-267, 2009.

[19] T. Kim, L.-C. Jang, and H. K. Pak, “A note on q-Euler and Genocchi numbers," Proceedings of the Japan Academy, Series A, vol. 77, no. 8, pp. 139-141, 2001.

[20] T. Kim, M.-S. Kim, L. Jang, and S.-H. Rim, "New q-Euler numbers and polynomials associated with p-adic q-integrals," Advanced Studies in Contemporary Mathematics, vol. 15, no. 2, pp. 243-252, 2007.

[21] S.-H. Rim and T. Kim, "A note on $p$-adic Euler measure on $\mathbb{Z}_{p}$, " Russian Journal of Mathematical Physics, vol. 13, no. 3, pp. 358-361, 2006.

[22] Y. Simsek, O. Yurekli, and V. Kurt, "On interpolation functions of the twisted generalized FrobeniusEuler numbers," Advanced Studies in Contemporary Mathematics, vol. 15, no. 2, pp. 187-194, 2007. 